\title{
CLINICAL EXPERIENCE WITH CARINAL RESECTION
}

John D. Mitchell, MD

Douglas J. Mathisen, MD

Cameron D. Wright, MD

John C. Wain, MD

Dean M. Donahue, MD

Ashby C. Moncure, MD

Hermes C. Grillo, MD
Objective: Pathologic processes that involve the carina pose a tremendous challenge to thoracic surgeons. Although techniques have been developed to allow primary resection and reconstruction, few institutions have accumulated sufficient experience to allow meaningful conclusions about the indications and the morbidity and mortality rates for this type of surgery. Methods: Since 1962, 135 patients have undergone 143 carinal resections (134 primary resection, 9 re-resection) at our institution. Indications for carinal resection included bronchogenic cancer (58 patients), other airway neoplasms (60 patients), and benign or inflammatory strictures (16 patients). Thirty-seven patients $(28 \%)$ had a history of prior lung or airway surgery not involving the carina. Carinal resection without pulmonary resection was accomplished in 52 patients; 57 patients had carinal pneumonectomy (44 right, 13 left); 14 patients had carinal plus lobar resection, and 11 patients had carinal resection after pneumonectomy ( 9 left, 2 right). There were 15 different modes of reconstruction, based on the type and extent of resection. Techniques were used to reduce anastomotic tension. Results: The operative mortality rate in the 134 patients after primary carinal resection was $12.7 \%$. Adult respiratory distress syndrome was responsible for 9 early deaths. Predominant predictors of operative death included postoperative mechanical ventilation $(P=.001)$, length of resected airway $(P=.03)$, and development of anastomotic complications $(P=.04)$. Mortality rates varied by the type of procedure and the indication for resection. Left carinal pneumonectomy was associated with a high operative mortality rate $(31 \%)$. Complications were noted in 52 patients $(39 \%)$, including atrial arrhythmias (20 patients) and pneumonia (11 patients). Anastomotic complications, both early and late, were seen in a total of 23 patients $(17 \%)$ and resulted in death or surgical reintervention in 21 patients $(91 \%)$. The operative mortality rate for carinal re-resection was $11.1 \%$. Conclusions: Carinal resection with primary reconstruction may be accomplished with acceptable mortality rates, but the underlying pathologic process and chance for long-term survival must be carefully considered before the operation is recommended, especially in the case of left carinal pneumonectomy. Anastomotic complications exact a heavy toll on involved patients. Careful patient selection and meticulous anesthetic and surgical technique remain the key to minimizing morbidity and mortality rates. (J Thorac Cardiovasc Surg 1999;117:39-53)
From the General Thoracic Surgical Unit, Massachusetts General Hospital, Department of Surgery, Harvard Medical School, Boston, Mass.

Read at the Seventy-eighth Annual Meeting of The American Association for Thoracic Surgery, Boston, Mass, May 3-6, 1998.

Received for publication May 8, 1998; revisions requested July 6, 1998; revisions received Sept 14, 1998; accepted for publication Sept 14, 1998.

\author{
Address for reprints: Douglas J. Mathisen, MD, Chief, General \\ Thoracic Surgery, Massachusetts General Hospital, Blake Bldg \\ 1570, Boston, MA 02114.
}

Copyright (C) 1999 by Mosby, Inc.

0022-5223/99 $\$ 8.00+0 \quad \mathbf{1 2 / 6 / 9 4 4 8 9}$ 
0 ver the past 20 years, reports of tracheal resection and sleeve lobectomy have well documented the indications and the morbidity and mortality risk of these categories of airway resection. Less is known about resection and reconstruction of the carina. Carinal resection remains a formidable challenge to thoracic surgeons, because of the problems posed by intraoperative airway management, the technical challenge of anatomic reconstruction, and the potential for significant postoperative morbidity. Previous reports of carinal resection, usually confined to right carinal pneumonectomy for bronchogenic carcinoma, described operative mortality figures ranging from $6.6 \%$ to $29 \% .^{1-4}$ Experience with carinal resection and reconstruction at the Massachusetts General Hospital dates to 1962 and was previously described by Grillo in $1982 .^{5}$ The present series is an update of that report and characterizes the indications and the morbidity, mortality, and risk factors of death for these procedures.

\section{Patients and methods}

All patients who underwent resection of the carina (with or without concomitant pulmonary resection) and primary reconstruction of the airway from 1962 through 1996 at the Massachusetts General Hospital were included in this study. Excluded were patients who underwent resection with staged reconstruction, a technique that was used occasionally early in our experience but that has since been abandoned because of the excessive morbidity and mortality rates associated with these procedures. Information was obtained from hospital charts, office records, referring physicians, and direct contact with the patients or patients' families.

Preoperative assessment. All patients scheduled to undergo carinal resection received a careful medical screening; special attention was paid to coexisting disease processes, cardiac and pulmonary function, and current medications. Complete pulmonary function tests, arterial blood gas analysis, and quantitative ventilation and perfusion scans were routinely performed to assess preoperative status and to predict postoperative lung function. In cases of concomitant pulmonary resection, a predicted postoperative forced expiratory volume in 1 second of $800 \mathrm{~mL}$ was used as a guide for resectability; allowances were made for patient size. An operation was not offered to patients whose condition was expected to require long-term mechanical ventilation. Because of the impact on anastomotic healing, long-term steroid therapy was considered a contraindication to operation, and attempts were made to wean patients from steroids 2 to 4 weeks before the resection. In cases of severe obstruction, patients could often be palliated with a bronchoscopic core-out as the steroids were weaned. ${ }^{6}$ A history of prior mediastinal irradiation, while no longer an absolute contraindication to operation, required special operative considerations including the use of an omental anastomotic wrap for most cases. ${ }^{7}$

Radiologic evaluation. All patients received computed tomography (CT) of the chest or tracheal/carinal linear tomograms or both. Chest CT allows for evaluation of the lung parenchyma, reveals mediastinal involvement either through identification of enlarged lymph nodes or direct extension of the disease process into adjacent structures, and aids in the metastatic work-up of carcinoma. Linear tomograms remain the most useful examination for assessing the extent of the disease process involving the airway and the amount of uninvolved airway remaining for reconstruction. Additional evaluation for metastases included CT of the head and bone scan, when indicated.

Anesthesia. An epidural catheter is currently placed before the operation to provide postoperative analgesia. An inhalation induction technique is used until the airway is secured. Anesthesia is maintained with inhalational agents and/or short-acting hypnotics, narcotics, and paralytic agents. Every effort is made to design an anesthetic plan that allows for extubation at the end of the procedure. An extra-long, flexible, armored single-lumen endotracheal tube is used, which may be advanced into a mainstem bronchus to provide one lung ventilation as indicated. Because of their size and inflexibility, double-lumen endotracheal tubes often present difficulties in these procedures. As the resection proceeds, the opposite (or remaining) mainstem bronchus is intubated across the operative field with a sterile endotracheal tube, with sterile connecting tubing passed off to the anesthesiologist. Close cooperation between the anesthesiologist and surgeon allows for intermittent ventilation of the lung alternating with temporary removal of the tube for precise placement of anastomotic sutures. As the end-to-end tracheobronchial anastomosis is brought together and the sutures are tied, the original endotracheal tube is advanced into the bronchus, and ventilation can proceed uninterrupted. The secondary anastomoses (eg, end of bronchus to side of trachea) can then be completed. It is important that the anesthesiologist be knowledgeable in the steps of the surgical procedure and be well versed in optional ventilatory techniques, including high frequency (jet) ventilation and independent lung ventilation. ${ }^{8}$

In our experience, cardiopulmonary bypass techniques are not needed for support during carinal resection and reconstruction in adults and older children. Cardiopulmonary bypass was used twice in this series. One patient underwent tangential excision of the main pulmonary artery (for invasive adenocystic carcinoma) with brief use of cardiopulmonary bypass. Bypass was also used early in this series to support a patient during a complex carinal reconstruction; severe pulmonary hemorrhage developed during the operation and was responsible for the only intraoperative death in the series.

Incision. In general, access to the carina is best achieved through a right posterolateral thoracotomy through the fourth or fifth intercostal space. For left carinal pneumonectomy, a left thoracotomy approach is used for limited resections, and either a bilateral thoracotomy or "clamshell" (bilateral submammary, transsternal) incision through the fourth intercostal space for more extensive resections. These latter approaches must be reserved only for the fittest patients. The anterior neck, chest, and ipsilateral arm are included in the 


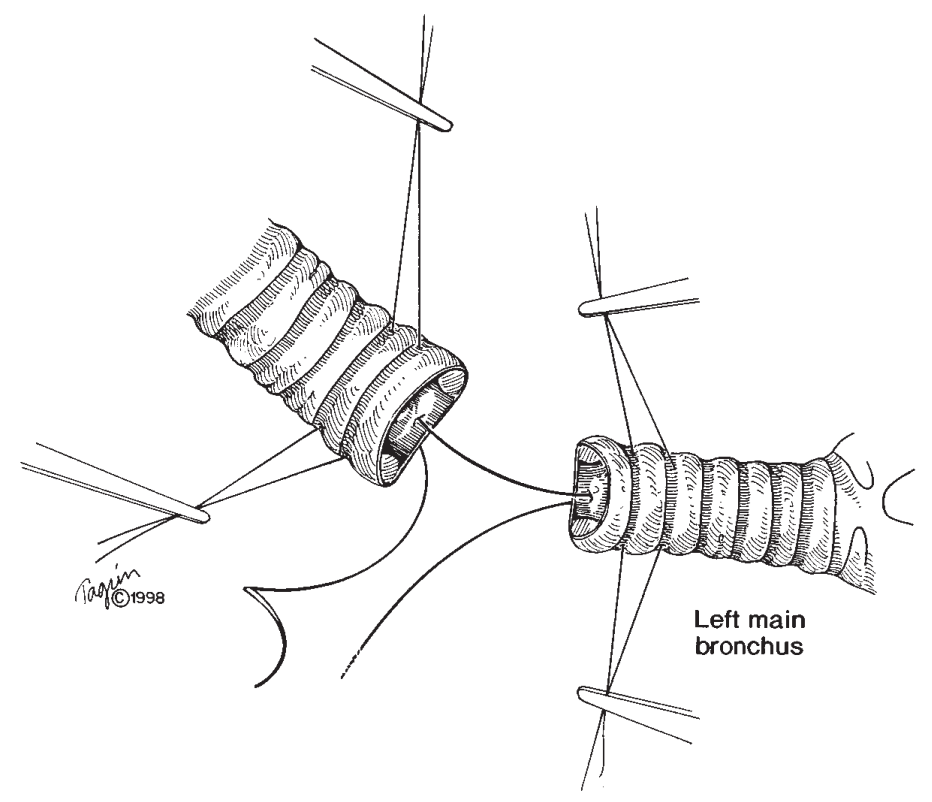

Fig 1. Technique of end-to-end anastomosis.

operative field should additional release maneuvers (typically, suprahyoid release) be needed. Aside from one case, median sternotomy was not used, because access is poor except for the simplest type of carinal reconstruction.

Assessment of resectability. All patients are examined with a rigid bronchoscope for precise visual assessment. When indicated, mediastinoscopy is performed to help assess whether resection is advisable or even feasible. The principle use of mediastinoscopy is in staging bronchogenic carcinoma involving the carina. Beyond the assessment of nodal status, mediastinoscopy is potentially useful for several reasons. First, the extent of the extraluminal spread of a malignant process involving the carina can be determined. In addition, complete development of the pretracheal plane is accomplished, improving mobility of the upper airway and lessening the chance of subsequent injury to the left recurrent laryngeal nerve when the distal trachea is dissected free at thoracotomy. Mediastinoscopy should be performed at the time of proposed resection, because prior mediastinoscopy may interfere with later tracheal mobility and make definition of the extent of the tumor and surgical planes difficult. Final assessment of resectability is completed at the time of thoracotomy. Limited involvement of the superior vena cava or esophagus (usually muscular wall) by tumor does not preclude resection if primary repair of the involved mediastinal structure can be accomplished.

Maneuvers to reduce anastomotic tension. Special care is taken to minimize tension on all airway anastomoses. Development of the pretracheal plane from below (if not performed at mediastinoscopy) without interrupting the lateral blood supply provides some mobility to the trachea. This same technique also supplies limited mobility when applied to the left main bronchus.
Significant reduction in anastomotic tension is provided by the mobilization of the hilum with an inferior hilar release on either side. ${ }^{5}$ Surgical approaches make it difficult to release both hila in a given case. The release is best accomplished before airway resection is begun. After the division of the inferior pulmonary ligament, a U-shaped incision is made in the pericardium beneath the hilum, with intrapericardial division of the raphe extending between the inferior pulmonary vein and the inferior vena cava. Even more hilar mobility can be achieved with a complete release, encircling the hilum with the pericardial incision. In a complete hilar release, care is taken to preserve the posterior lymphatic and vascular attachments to the hilum, by working beneath these structures. This type of release provides for cephalad displacement of the hilar structures over several centimeters and is particularly useful in cases of carinal resection where reimplantation of the right main bronchus or bronchus intermedius into the side of the trachea is planned.

Suprahyoid laryngeal release as described by Montgomery 9 was performed occasionally early in this series but was found not to be useful for carinal resection, unless the resection reaches the midtrachea. The effect of the laryngeal release did not translate to more distal tracheal mobility. Flexion of the neck devolves the cervical trachea toward the carina and is performed at the time that the end-to-end tracheobronchial anastomosis is brought together and secured. This maneuver is very important and may be easily overlooked. Some degree of cervical flexion is maintained beyond the end of the procedure with the use of a heavy stitch between the chin and the anterior chest wall. More important, this stitch prevents neck extension, which could disrupt the anastomosis, in the early postoperative period. Extreme flexion is to be avoided. This stitch is removed on postoperative day 7 .

Technique of carinal resection. The details of carinal 


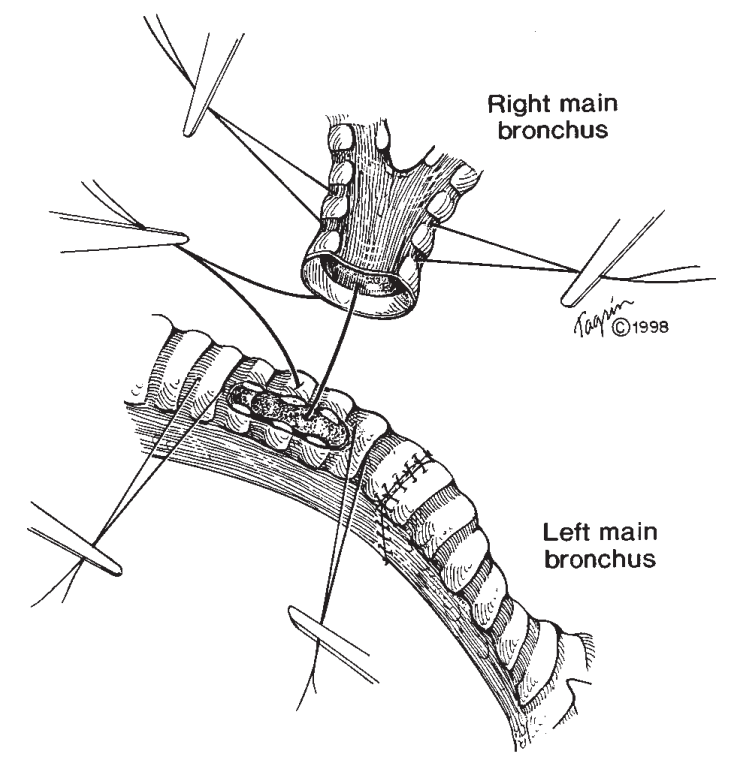

Fig 2. Technique of end-to-side anastomosis. Note that the created opening is entirely in cartilaginous wall, to provide rigidity to the anastomosis.

resection and reconstruction have been described previously. ${ }^{5}$ After the development of the pretracheal plane from below, tapes are placed around the mobilized trachea and left and right main bronchi. Care must be taken in encircling the trachea to avoid injuring the left recurrent laryngeal nerve. Except for a limited margin necessary for anastomosis, dissection of the airway must be limited to the planned area of resection, to avoid devascularization. Lymphadenectomy beyond removal of immediately adjacent or clearly involved nodes is avoided in a similar manner. When resection for a malignant process is planned, frozen section analysis of resected airway margins by competent pathologists must be available.

Once resection of the carina has been completed, ventilation continues as described earlier. Assessment is made regarding the optimal mode of reconstruction. This requires mature intraoperative judgment and is largely dependent on the relative extent of the trachea, left main bronchus, and right main bronchus resected. End-to-end airway anastomoses are begun by placement of traction sutures (2-0 Vicryl; Ethicon, Inc, Somerville, NJ) laterally both above and below the anastomosis. These traction sutures are tied across the anastomosis when the airway is brought together, reducing anastomotic tension. No tailoring of either airway is performed to account for discrepancies in size. Simple interrupted anastomotic sutures of 4-0 Vicryl polyglactin 910 are placed in a concentric fashion 3 to $4 \mathrm{~mm}$ apart, 3 to $4 \mathrm{~mm}$ from the cut edge of the airway, such that the knots will lie outside the airway when tied (Fig 1). Care is taken to avoid traumatizing the mucosa of the airway. When the placement of the sutures is completed, the indwelling endotracheal tube is advanced under direct vision into the bronchus beyond the anastomosis. After the traction sutures are secured, the anastomotic sutures are tied. The anastomosis is then tested for air leaks and repaired if needed; if proper care is taken during the original placement of sutures, the need for additional sutures is unusual.

End-to-side anastomoses are constructed in a similar manner, with 2 additional caveats (Fig 2). First, the ovoid opening created in the side of the trachea or bronchus should be entirely in cartilage, avoiding the membranous wall. This provides additional rigidity at the level of the anastomosis. Second, the opening should be at least $1 \mathrm{~cm}$ away from the end-to-end anastomosis, to avoid devascularization and necrosis of the intervening cartilaginous isthmus.

After the completion and testing of all suture lines, a circumferential wrap of each anastomosis is performed with viable tissue. This buttresses the anastomosis and separates the anastomosis from adjacent pulmonary vessels. Pleura was used primarily early in this series. A pedicled pericardial fat pad supplied by a branch of the internal thoracic artery is now preferred. Occasionally intercostal muscle and, particularly in cases where preoperative radiation was administered, omentum is used. Care must be taken to avoid leaving periosteum attached to circumferentially wrapped intercostal muscle pedicles; in one patient in this series, a bony grommet formed about the anastomosis after intercostal muscle use, resulting in stenosis requiring carinal re-resection.

Reconstruction of the carina. A variety of reconstructive options are available. For limited resections of the carina, the right and left mainstem bronchi can be reapproximated to form a "neocarina," which is then attached to the distal trachea (Fig 3). Cephalad movement of the newly formed carina is limited by the aortic arch, so most of the airway mobility in this reconstruction must come from the trachea. When a somewhat longer section of trachea is resected with the carina, precluding use of a "neocarina," the preferred mode of reconstruction involves end-to-end anastomosis of the trachea to left main bronchus, with reimplantation of the right main bronchus into the side of the trachea (Fig 4). This latter anastomosis is facilitated by right hilar release. If the gap (that is, the length of resected airway) between the trachea and left main bronchus exceeds $4 \mathrm{~cm}$, excessive anastomotic tension may result with this reconstruction, even after the use of release maneuvers. The reverse of this reconstructive option (trachea end-to-end with right main bronchus, left main bronchus into side of trachea) was used just once in this series, in part because of difficulties with exposure of the end-to-side anastomosis. For resection of the carina with extensive tracheal involvement, end-to-end anastomosis between the trachea and right main bronchus (mobilized with hilar release) and reimplantation of the left main bronchus into the side of the bronchus intermedius was preferentially used (Fig 5).

Occasionally, tumors or other disease processes involve primarily either the right or left mainstem bronchus with minimal involvement of the carinal spur. In these cases, it is possible to perform a nonsegmental resection of the affected bronchus and spur alone, maintaining continuity between the trachea and 

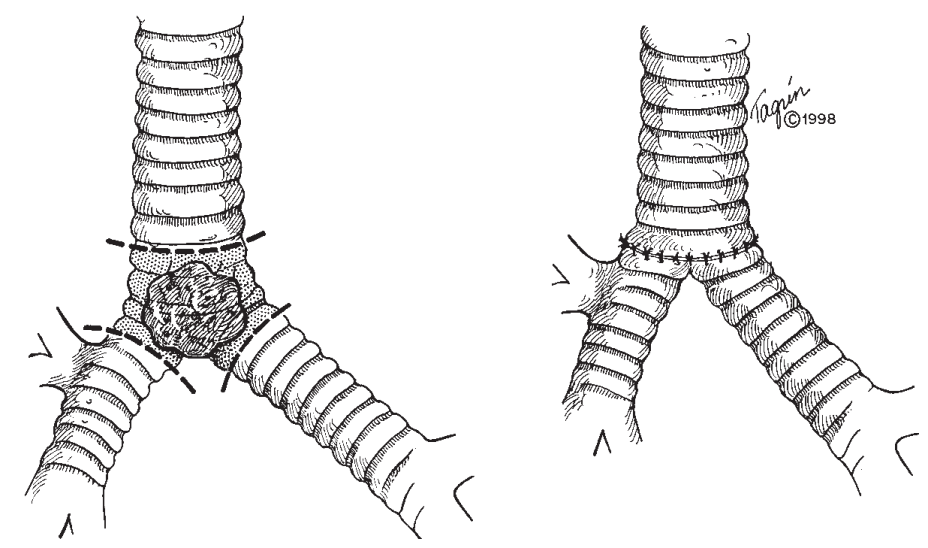

Fig 3. Resection with formation of a "neocarina." This technique is applicable for small, centrally placed tumors where only a limited resection is required.

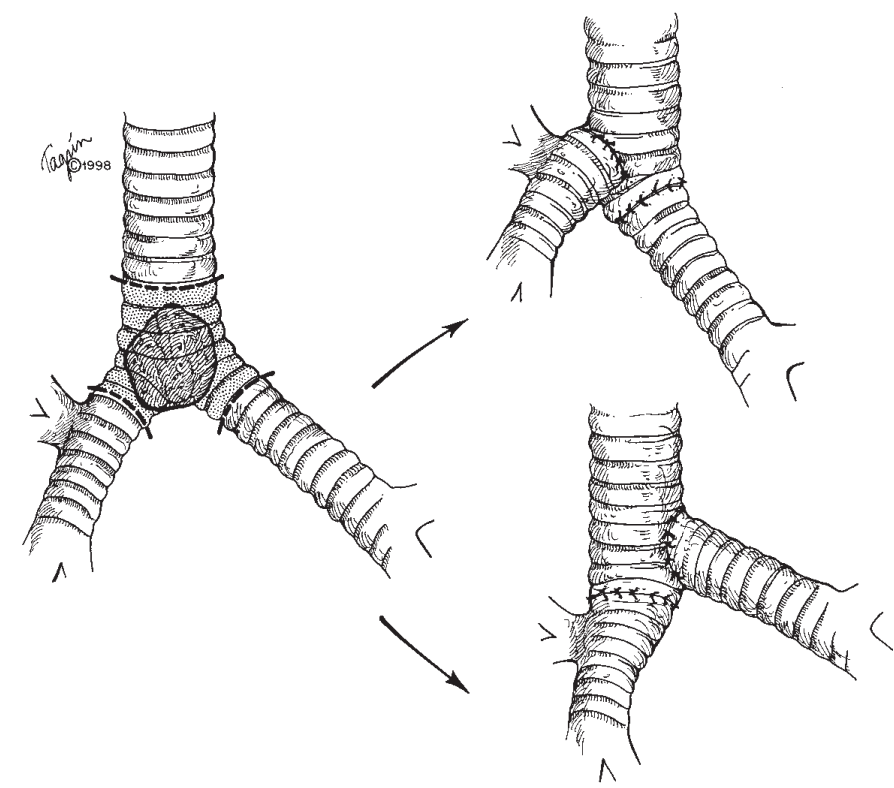

Fig 4. Carinal reconstruction with a moderate amount of airway resected. In these cases, the trachea may be anastomosed end to end with either the right or left mainstem bronchus, with the contralateral bronchus reimplanted into the side of the trachea. The diagram at the upper right shows the more commonly used technique.

contralateral bronchus. The detached bronchus is then sewn directly to the tracheobronchial defect caused by the resection. Tailoring because of size discrepancy is rarely needed.

If disease processes at the carina also involve lobar orifices (typically, right upper or middle lobes), resection can be accomplished that includes contiguous resection of the affected lobes (Fig 6). Reconstruction is complicated by the extensive length of resected airway, often making it difficult to achieve tension-free anastomoses. With resection of the carina and right upper lobe, the bronchus intermedius can sometimes be reimplanted to the side of the trachea with proper hilar mobilization but is more safely reimplanted in the left main bronchus. Advancing the bronchus intermedius to the level of the trachea for anastomosis can lead to airway necrosis and narrowing, caused by anastomotic angulation, devascularization, and tension. When the right middle lobe is included in the resection, the retained right lower lobe bronchus is best reimplanted into the side of the left main bronchus.

Extensive endobronchial involvement, destroyed lung as the result of bronchial obstruction, or involvement of the ipsilateral hilar vessels may mandate carinal (sleeve) pneumonectomy. This is particularly true in cases of bronchogenic carcinoma, where fear of missed nodal metastases can also favor 


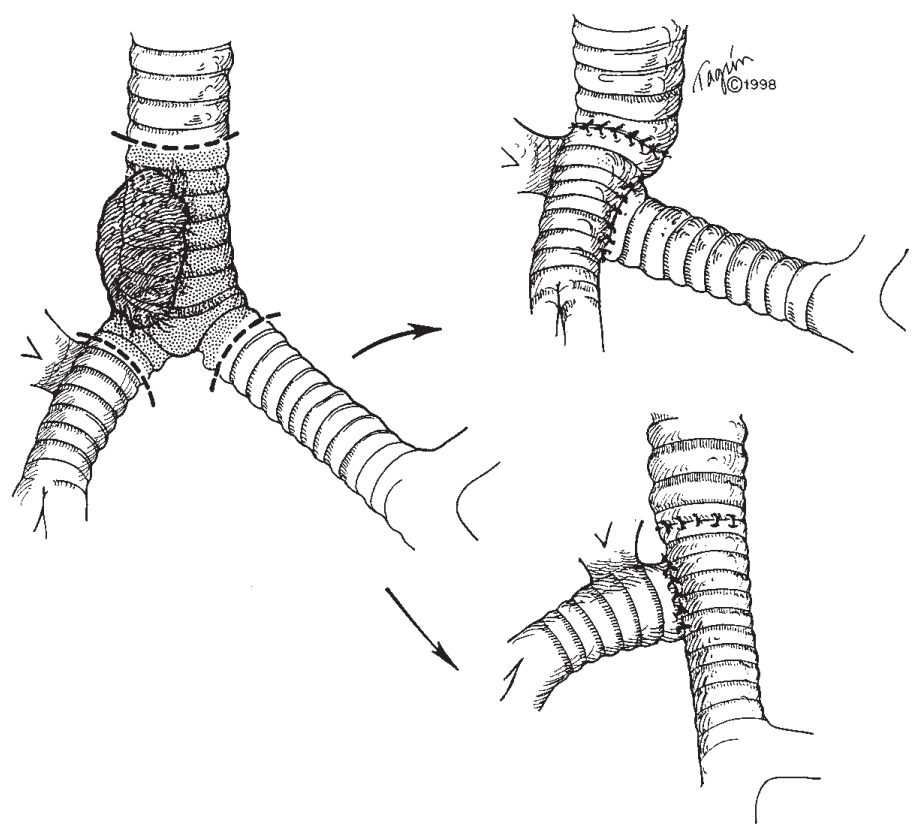

Fig 5. Carinal reconstruction with an extensive amount of airway resected. In these cases, the right mainstem bronchus is preferentially brought cephalad to the trachea for end-to-end anastomosis, with the left bronchus reimplanted into the bronchus intermedius (upper right diagram). This reconstruction is greatly facilitated by a right hilar release. The reverse procedure (lower right diagram) is rarely performed due to the immobility of the left main bronchus.

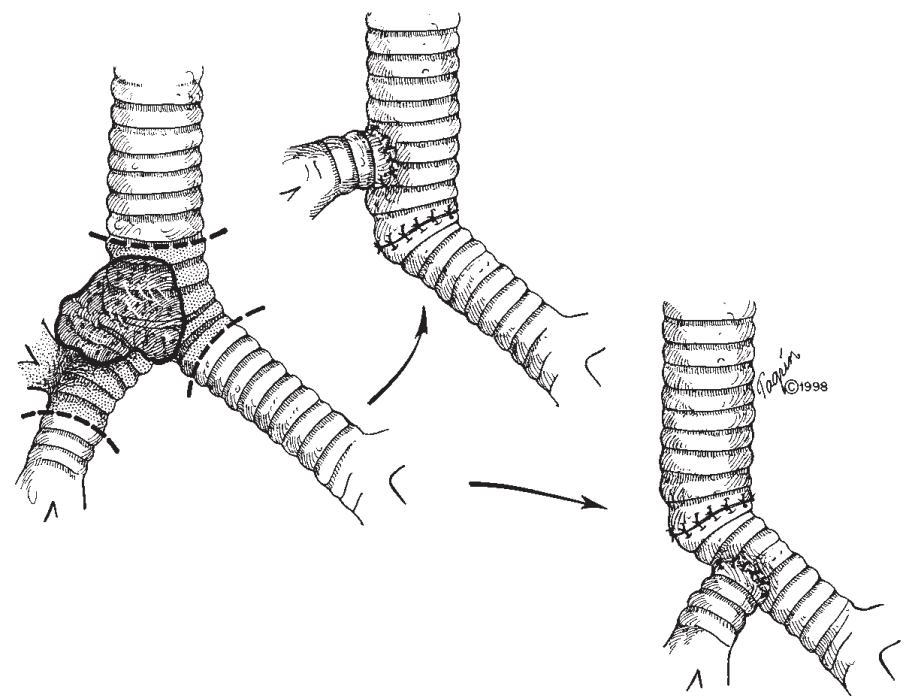

Fig 6. Carinal resection plus resection of the right upper lobe. After end-to-end anastomosis between the trachea and left mainstem bronchus, the bronchus intermedius is reimplanted into either the trachea (upper right diagram) or left mainstem (lower right diagram). Although this method was used more frequently in this series, significant morbidity has been noted with the trachea-bronchus intermedius anastomosis, presumably because of latent tension on the anastomosis.

pneumonectomy. Carinal pneumonectomy is achieved with end-to-end anastomosis of the trachea to the remaining mainstem bronchus (Fig 7). In the case of right carinal pneumonectomy, difficulties may arise because of the relative immobility of the left main bronchus. With left carinal pneu- monectomy, particularly from a left-sided approach, exposure can be difficult because of the aortic arch. Exposure can be improved with mobilization of the aorta and either a clamshell or bilateral thoracotomy approach, but only at physiologic cost to the patient. 

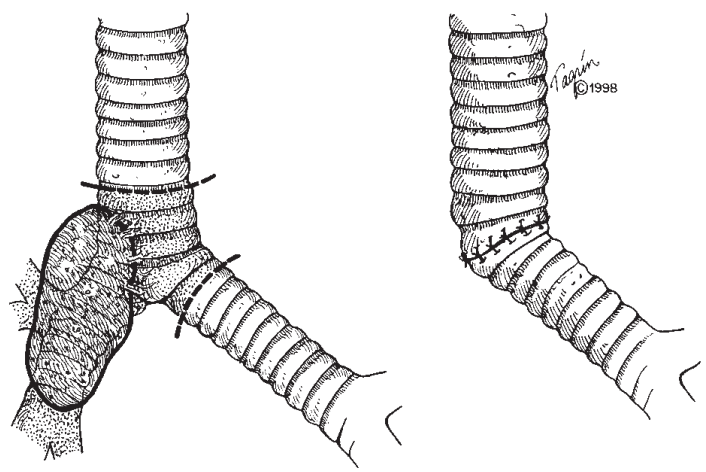

Fig 7. Carinal (sleeve) pneumonectomy. The trachea and contralateral (in this case, left) bronchus are brought together in an end-to-end anastomosis.

Carinal resection after prior pneumonectomy (Fig 8) is achieved with end-to-end reconstruction similar to carinal pneumonectomy. A right-sided approach is preferred, although the procedure may be performed through a leftsided incision if a specific indication exists. Care is used to avoid injury to the often heavily scarred pulmonary artery stump. After prior left pneumonectomy, a right-sided approach is performed with gentle retraction of the inflated right lung, sometimes working in intermittent fashion.

Statistical methods. Predictors of postoperative death were identified through multiple logistic regression analysis with the use of the computer software program STATA (Stata Corporation, College Station, Texas).

\section{Results}

From 1962 through 1996, 134 patients (70 male patients, 64 female patients) underwent resection of the carina with primary (single-stage) reconstruction of the airway. One half of these patients (67) underwent reconstruction within the last 9 years. The mean age was 47.8 years (range, $8-80 \mathrm{yr}$ ). The mean age varied with diagnosis: Patients with bronchogenic carcinoma were older as a group (mean age, 55.8 years; range, 8-73 yr) than those patients with other airway neoplasms (mean age, 40.2 years; range, 8-67 years) or with benign airway strictures (mean age, 48.2 years; range, 18-80 years).

Prior operation. Thirty-seven patients $(28 \%)$ had a history of lung or airway operation. This included 11 patients who had undergone pneumonectomy (9 left, 2 right), 14 patients who had undergone a lesser lung resection (9 lobectomy or bilobectomy, 5 wedge resection), and 9 patients who had previously undergone transthoracic tracheotomy and localized tumor resection. Two patients had a history of tracheal resection and reconstruction for carcinoma, with positive resection margins. One patient had had a laryngectomy 7
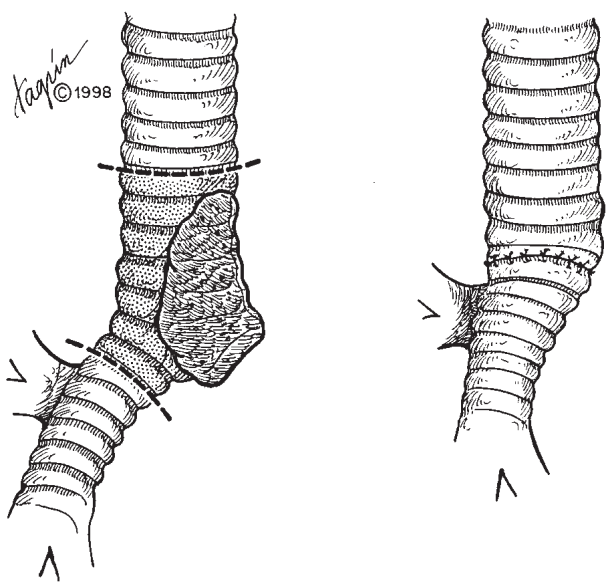

Fig 8. Resection of the carina after pneumonectomy. These procedures are usually completed through a right thoracotomy approach, regardless of the side of previous pneumonectomy.

years before the squamous cell carcinoma and a right upper lobectomy 2 years previously for the same histologic reasons. He underwent right carinal (completion) pneumonectomy for recurrent carcinoma arising from the right upper lobe stump encroaching on the carina.

Diagnosis. The indications for carinal resection with primary reconstruction are listed in Table I. One patient, who had undergone right upper lobectomy 1 year previously for non-small cell carcinoma, had a new, very limited carinal lesion. Biopsy yielded small cell carcinoma. After a negative metastatic evaluation and mediastinoscopy, he underwent successful carinal resection and reconstruction with postoperative adjuvant chemotherapy and radiotherapy. Subsequent review of his original right upper lobe pathologic evidence revealed a small focus of small cell carcinoma similar to his carinal lesion. He remains disease-free now 5 years after the resection.

The most diverse group of patients were those with benign or inflammatory diseases of the carina. Three patients had idiopathic circumferential fibrosis of the lower trachea, carina, and mainstem bronchi, similar to that previously reported involving the larynx and upper trachea. ${ }^{10}$ Two patients had extreme mediastinal shift and tracheobronchial malacia after prior pneumonectomy, or "postpneumonectomy syndrome."11 These individuals required other procedures (mediastinal repositioning, aortopexy) in addition to airway resection to prevent recurrent tracheomalacia. In spite of these maneuvers, one of these patients required re-resection. Another patient underwent a left main bronchial resection 3 years previously at an outside institution for a plasma cell tumor, during which the right main 
Table I. Diagnosis in primary carinal resection and reconstruction $(n=134)$

\begin{tabular}{lr}
\hline Bronchogenic carcinoma $(\mathrm{n}=58)$ & 42 \\
Squamous cell carcinoma & 10 \\
Adenocarcinoma & 4 \\
Large cell carcinoma & 1 \\
Small cell carcinoma & 1 \\
Bronchoalveolar carcinoma & \\
Other airway neoplasms $(\mathrm{n}=60)$ & 37 \\
Adenoid cystic carcinoma & 11 \\
Carcinoid & 7 \\
Mucoepidermoid carcinoma & 2 \\
Malignant fibrosing histiocytoma & 1 \\
Fibrosarcoma & 1 \\
Mixed spindle cell carcinoma & 1 \\
Granular cell tumor & \\
Benign/inflammatory structures $(\mathrm{n}=16)$ & 5 \\
Fibrosing mediastinitis & 3 \\
Idiopathic & 2 \\
Postpneumonectomy syndrome & 2 \\
Tubercular stenosis & 1 \\
Ischemic/iatrogenic stenosis & 1 \\
Mucormycosis & 1 \\
Amyloidoma & 1 \\
Hamartoma &
\end{tabular}

bronchus was injured and was primarily repaired. Unfortunately, the repair and the left main bronchial anastomosis broke down in the early postoperative period, and she underwent subsequent left pneumonectomy and re-repair of the right main bronchus. Over the ensuing 3 years she experienced the development of progressive "asthma" symptoms, culminating in respiratory failure, intubation, and discovery of a tight $(2 \times$ $7 \mathrm{~mm}$ ) stenosis of the right main bronchus. She was transported to our hospital supported by mechanical ventilation and was treated successfully by resection and reconstruction.

Preoperative evaluation and therapy. All patients underwent preoperative bronchoscopy. Twenty-five patients (18\%) with significant malignant airway obstruction required preoperative endoscopic debulking of the tumor, typically accomplished with the use of the rigid bronchoscope. This procedure was performed when respiratory failure as the result of airway obstruction was present, to assist in the management of postobstructive pneumonia before resection, and to facilitate airway access at the time of carinal reconstruction. Preoperative mediastinoscopy was performed in 48 patients. Ten patients had been treated with preoperative radiotherapy: 4 of these patients were treated with concomitant chemotherapy as part of a neoadjuvant protocol; 3 patients were given radiotherapy after lung resection for bronchogenic carcinoma, and 3 patients were initially treated with radiation at an outside facil- ity before being referred for what was presumed to be an inoperable lesion.

Incision. Excluding patients whose condition required left carinal pneumonectomy, a right posterolateral thoracotomy approach was used in 92\% (111 of $121)$ of cases. Three patients with a history of pneumonectomy underwent carinal resection through a left thoracotomy. In 2 patients, a left-sided approach was used for partial resection of the carina with reconstruction for lesions primarily involving the left main bronchus. In 2 cases of carinal resection or extensive adenoidcystic carcinoma, a clamshell approach through the fourth intercostal space was used, allowing for bilateral hilar mobilization. Similarly, 1 patient underwent right carinal pneumonectomy through a median sternotomy approach to allow for left hilar release if needed. Early in this series, 2 patients underwent carinal resection through right thoracotomy with cervical/sternal extension.

For patients whose condition required left carinal pneumonectomy, a left posterolateral thoracotomy incision was used in 5 patients when it was estimated the extent of airway resection (trachea to right main bronchus) would be limited. For other patients for whom a more extensive resection was envisioned, either a clamshell (5 cases) or a bilateral thoracotomy ( 3 cases) approach was used.

In the 10 patients who underwent resection with either a bilateral thoracotomy or a clamshell incision, 6 patients required postoperative ventilatory assistance.

Operative procedure. Carinal resection and reconstruction without pulmonary resection was accomplished in 52 patients. Carinal pneumonectomy was performed in 57 patients (44 right, 13 left). Fourteen patients underwent resection of the carina plus either the right upper lobe (11 cases) or both the right upper and middle lobes ( 3 cases). Eleven patients underwent resection of the carina after previous pneumonectomy (9 left, 2 right).

The average extent of airway resection (total length of airway measured from tracheal line of resection to the divided mainstem bronchus used for the end-to-end tracheobronchial anastomosis) was $2.9 \mathrm{~cm}$. (range, 1-6 $\mathrm{cm})$. The average length of resected airway for those patients who had an anastomotic complication or postoperative death was $3.7 \mathrm{~cm}$ and $4.0 \mathrm{~cm}$, respectively. The extent of resected airway was clearly related to development of an anastomotic problem for those patients (89 in all) with a trachea-left main bronchial anastomosis. In this group, the incidence of an anastomotic complication rose from $10 \%$ if the resection was limited to less than $4 \mathrm{~cm}$ and to $32 \%$ if 4 or more $\mathrm{cm}$ of airway were removed. 


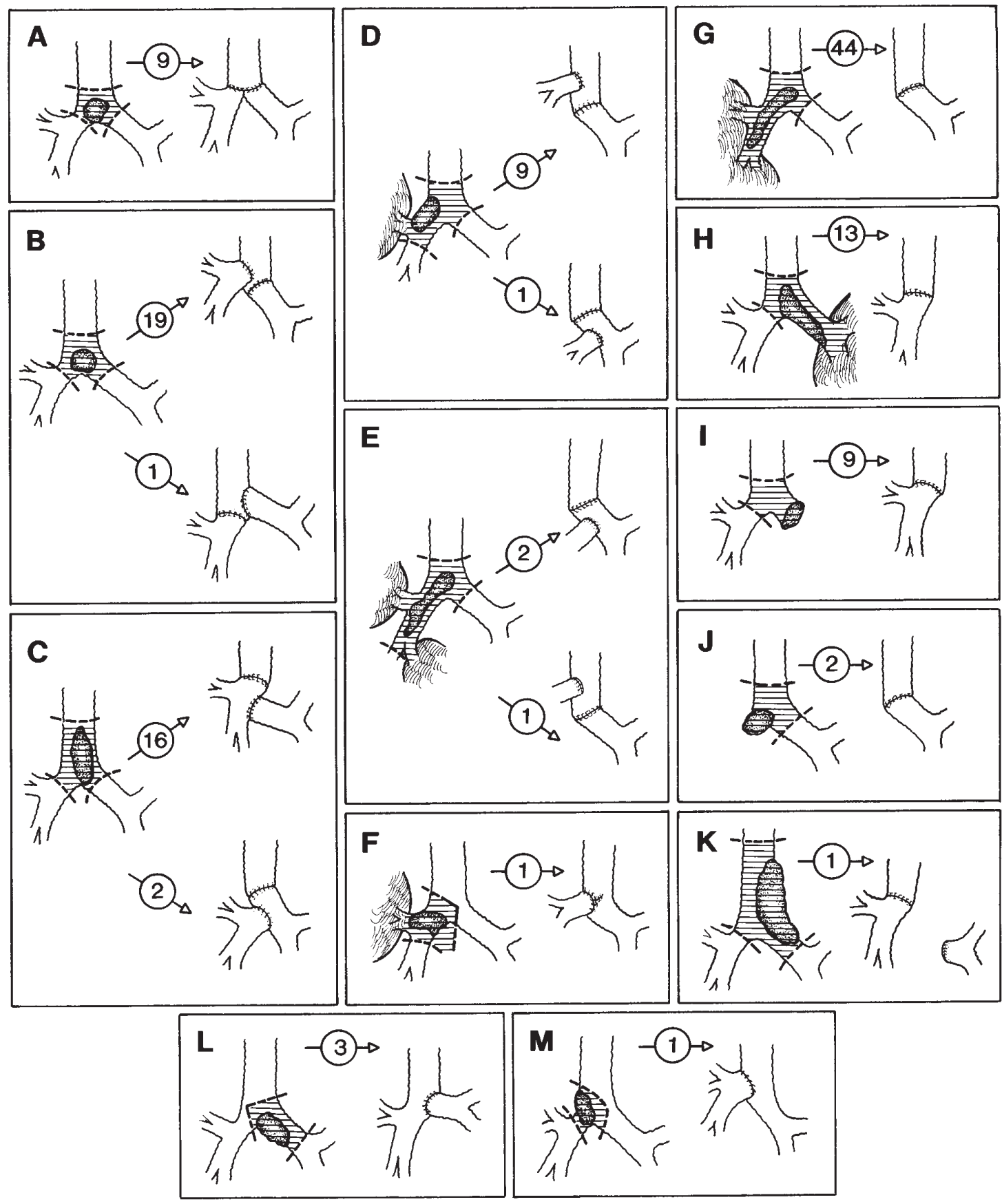

Fig 9. The various types of carinal reconstruction. The numbers in the circles represent the number of cases with each reconstruction. A-C, Carinal resection without concomitant pulmonary resection. D-F, Carinal plus lobar resection. G, Right carinal pneumonectomy. H, Left carinal pneumonectomy. I-J, Carinal resection after previous pneumonectomy. K, Carinal resection and reconstruction with left lung exclusion. L-M, Partial carinal resection and reconstruction.

Beyond resection and reconstruction of the airway, 21 patients underwent an additional intrathoracic procedure. Most of these involved malignant invasion of an adjacent mediastinal structure, requiring repair of the superior vena cava (10 cases), esophagus (6 cases), or both (1 case). One patient with adenocystic carcinoma of the left main bronchus and carina had invasion of the main pulmonary artery that required partial resec- 
Table II. Morbidity/mortality rates in primary carinal resection and reconstruction

\begin{tabular}{|c|c|c|c|c|}
\hline Procedure or diagnosis & $n$ & $\begin{array}{c}\text { Operative deaths } \\
\text { (\%) }\end{array}$ & $\begin{array}{l}\text { Operative morbidity } \\
(\%)\end{array}$ & $\begin{array}{c}\text { Anastomotic } \\
\text { complications } \\
(\%)^{*}\end{array}$ \\
\hline Carinal resection & 52 & $4(7.7)$ & $14(26.9)$ & $8(15.4)$ \\
\hline Right carinal pneumonectomy & 44 & $7(15.9)$ & $18(40.9)$ & $3(6.8)$ \\
\hline Left carinal pneumonectomy & 13 & $4(30.8)$ & $9(69.2)$ & $5(38.4)$ \\
\hline Carinal plus lobar resection & 14 & $1(7.1)$ & $7(50)$ & $5(35.7)$ \\
\hline Carinal resection after pneumonectomy & 11 & $1(9.1)$ & $4(36.4)$ & $2(18.2)$ \\
\hline Bronchogenic carcinoma & 58 & $9(15.5)$ & 27 (46.6) & $11(19.0)$ \\
\hline Other airway neoplasms & 60 & $5(8.3)$ & $17(28.3)$ & $10(16.7)$ \\
\hline Benign/inflammatory lesions & 16 & $3(18.7)$ & $8(50)$ & $3(18.7)$ \\
\hline Total & 134 & $17(12.7)$ & $52(38.8)$ & $23(17.2)$ \\
\hline
\end{tabular}

*Includes both early and late anastomotic morbidity.

Table III. Predictors of postoperative death in primary carinal resection and reconstruction

\begin{tabular}{lcccc}
\hline & & \multicolumn{2}{c}{$95 \%$ Confidence limits } & Upper \\
\cline { 3 - 4 } Variables & Odds ratio & Lower & 73.56 & .001 \\
\hline Postoperative mechanical ventilation & 14.61 & 2.90 & 4.46 & .03 \\
Extent of resected airway & 2.18 & 1.06 & 46.49 & .04 \\
Anastomotic morbidity & 7.07 & 1.08 & 30.63 & .08 \\
Additional intrathoracic procedure & 5.01 & 0.82 & 59.25 & .11 \\
Preoperative radiation & 6.18 & 0.64 & 14.62 & .27 \\
Concomitant lung resection & 2.64 & 0.48 & 2.14 & .27 \\
Sex & 0.38 & 0.07 & 14.80 & .28 \\
Previous lung or tracheal operation & 2.62 & 0.46 & 1.05 & .99 \\
Age & 1.00 & 0.96 &
\end{tabular}

Overall model, $P<.001$.

Coefficient of determination, 0.45 .

tion and repair with a pericardial patch under brief cardiopulmonary bypass support. One patient with bronchoalveolar carcinoma had a necrotic, abscessed right lung and a tracheoesophageal fistula involving the carina after receiving an experimental bronchial artery infusion of chemotherapeutic drugs at another institution. In addition to right carinal pneumonectomy, she underwent successful closure of the fistula.

Additional maneuvers to minimize anastomotic tension were performed in 64 patients, consisting of a hilar release (49 cases), laryngeal release ( 3 cases), or both (12 cases). Nearly all anastomoses were wrapped with healthy, autogenous tissue, primarily either pleura or a pedicled pericardial fat pad. Omentum was used in 5 cases where preoperative radiotherapy was given and in the 1 case of tracheoesophageal fistula mentioned earlier where widespread mediastinitis was present.

Airway reconstruction. A number of different modes of reconstruction were used. A schematic representation of the various modes and their frequency of use are detailed in Fig 9.
Postoperative outcome. Nineteen patients (14\%) required mechanical ventilation after the operative procedure. The median length of hospital stay after the operation was 10 days (range, 5-120 days). The operative mortality, total morbidity, and anastomotic morbidity rates are detailed in Table II, grouped by procedure category and diagnosis. The mortality rate for carinal pneumonectomy, either right $(15.9 \%)$ or left $(30.8 \%)$, was substantially higher than for other procedures. The operative mortality rate for the initial 67 patients (one half of the patient cohort) was $16.1 \%$, compared with $9.0 \%$ for the last 67 patients. Adult respiratory distress syndrome (ARDS) was seen in 10 patients, predominately after carinal pneumonectomy (8 of 10 patients). ARDS was associated with a mortality rate of $90 \%$. There were 13 early deaths (0-33 days) primarily because of ARDS and 4 late deaths (75-120 days), all related to anastomotic complications. The predominant risk factors for postoperative death identified by logistic regression analysis included postoperative mechanical ventilation, extent of resected airway, 
and development of anastomotic complications (Table III).

Anastomotic morbidity was most prevalent after left carinal pneumonectomy (38\%) and carinal plus lobar resection (36\%). In this latter group, $80 \%$ of the patients in whom anastomotic problems developed were seen beyond the initial postoperative period.

Complications after carinal resection are listed in Table IV. The diagnosis of ARDS was made in the setting of diffuse pulmonary infiltrates, nonpurulent sputum, unremarkable sputum culture results, and no clinical evidence of left atrial hypertension. Cardiac herniation through a pericardial defect occurred in 1 patient 1 hour after a right carinal pneumonectomy with intrapericardial dissection of vessels and was repaired with a prosthetic patch. One woman had recurrent adenocystic carcinoma after a previous transthoracic excision of tumor. After a left carinal pneumonectomy through a bilateral thoracotomy approach, a dense sensorimotor deficit below the $\mathrm{C}_{5-6}$ level developed. The cause of this devastating complication was unclear.

Further procedures. Sixteen patients experienced complications requiring re-intervention after the initial procedure. Anastomotic complications in 7 patients required carinal re-resection. Two patients experienced the development of recurrent right lung infections, culminating in abscess formation after initial carinal resection and right upper lobectomy (bronchus intermedius implanted into the trachea). They were treated successfully with completion pneumonectomy. In another case, an empyema after right carinal pneumonectomy was treated successfully with thoracostomy and subsequent muscle transposition to facilitate cavity obliteration. One patient who underwent carinal resection and left lung exclusion early in the series required an interval left pneumonectomy for persistent hypoxemia. Two patients experienced fatal bronchovascular fistulas related to complications of anastomotic narrowing (stent erosion, 1 patient; abscess formation, 1 patient) several months after the initial operation. Another case of anastomotic stenosis after carinal resection was treated successfully with serial silicone T-Y and T tubes. One patient, who underwent reconstruction of a "neocarina" after extensive $(5 \mathrm{~cm})$ carinal resection, experienced an anastomotic separation that was treated successfully with silicone stenting, with ultimate removal of the stent. Recurrent rightsided pneumonias in another patient several years after the carinal resection after remote left pneumonectomy was attributed to postpneumonectomy syndrome, requiring mediastinal repositioning.
Table IV. Complications in primary carinal resection and reconstruction

\begin{tabular}{lcc}
\hline Complication & $n$ & Percent of 134 \\
\hline Anastomotic*† & 23 & 17.2 \\
Atrial arrhythmias & 20 & 14.9 \\
Pneumonia & 11 & 8.2 \\
ARDS & 10 & 7.5 \\
Reintubation $\ddagger$ & 3 & 2.2 \\
Vocal cord paresis & 3 & 2.2 \\
Gastrointestinal bleed & 3 & 2.2 \\
Empyema & 3 & 2.2 \\
Lobar collapse & 2 & 1.5 \\
Fever & 2 & 1.5 \\
Cardiac herniation & 1 & 0.8 \\
Phrenic nerve paresis & 1 & 0.8 \\
Candida esophagitis & 1 & 0.8 \\
Arterial embolus & 1 & 0.8 \\
Aspiration & 1 & 0.8 \\
Quadriplegia & 1 & 0.8 \\
\hline
\end{tabular}

*Necrosis, separation, stenosis, bronchial mucosal slough, excessive granulation tissue formation.

$\dagger$ Includes early and late morbidity.

\$After respiratory failure not the result of pneumonia or ARDS.

Carinal re-resection. The conditions of 9 patients required re-resection of the carina. Eight patients had anastomotic stenosis or separation (7 patients from our institution; 1 patient underwent resection elsewhere), and 1 patient had a recurrence of adenocystic carcinoma 17 years after the initial resection. Excluding the case of recurrent cancer, the mean interval from initial procedure to re-resection was 11 months (range, 1.5-52 months). The median length of postoperative stay was 11 days (range, 7-36 days). One patient (11.1\%) died.

Impact of anastomotic morbidity. A total of 23 patients (17.1\%) experienced an anastomotic complication at some point after the carinal resection. These complications ranged from necrosis, separation, and mucosal slough (seen early) to stenosis, excessive granulation tissue, and recurrent episodes of obstructive pneumonia (seen late). The time of presentation varied from the immediate postoperative period up to 10 years after the original procedure. Eight of these patients died in the immediate postoperative period, and another 2 died of bronchovascular fistulae within the first year, culminating in an overall procedure-related mortality rate of $43.5 \%$ ( 10 of 23 patients) associated with the development of anastomotic problems. Further, of the 13 remaining patients, the conditions of 11 patients required a major operative intervention to address the area of concern (re-resection, 7; completion pneumonectomy, 2; prolonged airway stenting or repeated dilations, 2). 


\section{Discussion}

Despite sporadic reports in the literature over the past 50 years, carinal resection remains a relatively daunting and infrequently used procedure for most thoracic surgeons. This retrospective study constitutes the largest single series of carinal resection and reconstruction yet reported and attempts to better define the indications and techniques and the morbidity and mortality rates of these procedures. These latter data may be subject to criticism, given the long accrual period for this series (34 years). Indeed, the improvement in the mortality rate from the first half of the cohort $(16.1 \%)$ to the second half $(9.0 \%)$ can be explained not only by the application of lessons learned over time but also because of the enormous advances made in anesthetic and critical care management during the same time period. Nonetheless, our approach to carinal resection, including the anastomotic techniques and the reconstructive options, remains largely unchanged from those clearly outlined by Grillo ${ }^{5}$ nearly 2 decades ago.

The overall mortality rate in this study $(12.7 \%)$ is comparable to those figures reported in other series. ${ }^{1-5}$ The predominant predictors of postoperative death included postoperative mechanical ventilation, the extent of airway resection, and the development of anastomotic complications (Table III). Mechanical ventilation predisposes to barotrauma and the development of infectious pulmonary complications and is detrimental to airway healing. Postoperative ventilation has been previously shown as a risk factor for the development of bronchopleural fistula after pneumonectomy. ${ }^{12}$ In our study, $79 \%$ of patients whose condition required postoperative ventilation had significant morbidity. Every attempt should be made, through careful patient selection and meticulous surgical and anesthetic technique, to extubate these patients at the end of the procedure. This should be achievable in most patients.

Earlier reports of carinal surgery stressed that the extent of airway resection should be limited to less than $4 \mathrm{~cm}$ to minimize the risk of an anastomotic complication when the trachea is to be reconnected end to end with the left main bronchus. ${ }^{1,13}$ This is due primarily to the relative immobility of the left bronchus, which is tethered in its cephalad migration by the aortic arch. The lack of mobility may result in excessive tension on the anastomosis. These limitations are not applicable to anastomoses involving the trachea end to end with the right main bronchus, which can be mobilized extensively with a full right hilar release. In this study we confirmed the validity of this rule, noting that, in patients with a trachea-left main bronchial anastomosis, the incidence of anastomotic problems tripled when the extent of resection was $4 \mathrm{~cm}$ or larger. Techniques to reduce anastomotic tension should routinely be used. We use neck flexion, dissection of the pretracheal plane, traction sutures, and a postoperative chin stitch in all patients. Hilar release, either partial or full, should be used whenever indicated.

The impact of anastomotic complications cannot be underestimated in patients after carinal resection. Ninety-one percent of the patients in this series with anastomotic problems experienced either procedurerelated morbidity or death. Beyond the effects of postoperative ventilation and excessive anastomotic tension, errors in surgical technique can be major contributors to the development of anastomotic morbidity. Precise suture placement, avoidance of airway devascularization, and atraumatic handling of the airway mucosa are all key factors in the successful completion of anastomoses. For the end-to-side secondary anastomoses, the created airway opening should be entirely in cartilage to provide rigidity and should be at least $1 \mathrm{~cm}$ away from the end-to-end anastomosis to avoid intervening airway necrosis. We believe the use of an anastomotic wrap with healthy, viable tissue is helpful, both as anastomotic buttress and vascular buffer.

Beyond these primary predictors of death (postoperative ventilation, extent of resection, anastomotic problems) were less important risk factors, including the need for additional intrathoracic procedures $(P=.08)$ and, to a lesser extent, preoperative radiotherapy $(P=$ .11). The requirement of an additional procedure (typically resection of the vena cava or esophagus) may be construed as a marker for a more extensive resection. Although the numbers were small (10 cases), none of the patients who had received radiation in the past experienced an anastomotic complication. This may be due to the liberal use of an omental wrap in many of these cases. Interestingly, prior lung or airway operation was not a significant risk factor for operative death in this series. The mortality figure for carinal re-resection $(11.1 \%)$ further supports the fact that these procedures can be accomplished in a reoperative setting.

Several of the other statistics in Table II deserve mention. There was a marked difference in mortality rates between those patients with bronchogenic carcinoma $(15.5 \%)$ and other airway neoplasms $(8.3 \%)$, perhaps in part because of the age difference (and attendant comorbidity) between the 2 groups (mean age, 55.8 years vs 40.2 years). In addition, patients with bronchogenic carcinoma are more likely to have a smoking history and thus may have had compromised lung function compared with the group with other neoplasms. 
Finally, patients with bronchogenic carcinoma more commonly required carinal pneumonectomy (34 of 58 patients [59\%]) than those patients with other malignant diseases of the airway (16 of 60 patients [30\%]). The aggregate mortality rate for carinal pneumonectomy (right and left) in this series was $19.3 \%$ (11 of 57 patients). At least part of the increased mortality rate seen with carinal pneumonectomy can be attributed to the prevalence of ARDS in this group; 8 of the 10 patients who experienced the development of ARDS did so after carinal pneumonectomy.

Those patients with benign or inflammatory strictures represented a diverse group of diagnoses, and thus less can be concluded from the accompanying data. Many of the operations in this group were quite difficult because of diffuse airway involvement in the pathologic process (eg, idiopathic stenosis) or because of the involvement of surrounding mediastinal structures (eg, fibrosing mediastinitis). For several of these patients, an operation represented a last, heroic option to treat the illness. In the future, some of these lesions may be amenable to airway stenting.

Significant mortality (31\%), overall morbidity $(69 \%)$, and anastomotic morbidity $(38 \%)$ rates were associated with left carinal pneumonectomy. Several reasons may account for these statistics. First, the approach to the carina via a left thoracotomy is quite limited, primarily because of the aortic arch. Mobilization of the aorta is required for access to the carina. These difficulties in exposure make the dissection, mobilization, and subsequent anastomosis of the airway much more problematic, particularly when a more extensive resection is required. The exposure can be improved through either a bilateral thoracotomy or clamshell approach as was performed in 8 of 13 patients in this series, but at great physiologic cost to the individual; of the patients with these types of incisions, $60 \%$ required postoperative mechanical ventilation. Although infrequently performed, careful consideration of the underlying pathologic process and the chance for long-term survival must be made before this procedure is offered to selected patients.

Patients undergoing carinal plus lobar resection also had significant $(36 \%)$ anastomotic morbidity. This was almost invariably due to problems at the end-to-side secondary anastomosis, between the bronchus intermedius or right lower lobe bronchus and the trachea or left main bronchus. Despite meticulous attention to the factors mentioned earlier (tension, devascularization, and anastomotic technique), narrowing developed at the anastomosis, often leading to recurrent bouts of pneumonia, bronchiectasis, and eventual abscess in the re- tained right lung. These patients required re-resection or completion pneumonectomy, sometimes years after the initial operation. On the basis of this experience, we feel that in patients who require carinal plus lobar resection, consideration should be given to carinal pneumonectomy if the individual can otherwise tolerate such a procedure. If reimplantation is to be performed (either bronchus intermedius or right lower lobe bronchus), it should be to the side of the left main bronchus and not to the trachea. Anastomosis of these bronchi to the trachea creates excessive tension, unlike implantation of the right mainstem bronchus to the trachea.

We excluded those patients who underwent staged carinal resection and reconstruction, that is, where excessive airway resection required tracheal division with planned re-establishment of airway continuity at a later date. These procedures too often had a dismal outcome and thus are no longer performed. ${ }^{5}$ This fact underscores the importance of careful assessment before resection, to ascertain whether re-establishment of airway continuity can be accomplished. In some instances (particularly with adenocystic carcinoma) this may mean accepting a microscopic positive margin, relying on postoperative radiotherapy to sterilize the residual tumor foci. ${ }^{14}$

A successful outcome for carinal resection and reconstruction depends on many factors. Careful patient selection as to who can tolerate the physiologic effects of the operation cannot be underestimated. Understanding the safe limits of resection, the technical nuances of airway reconstruction, and methods to reduce anastomotic tension should minimize the serious problem of anastomotic morbidity which was associated with a $43 \%$ mortality rate in this series. Finally, an improved ability to treat ARDS will significantly impact the outcome of patients after carinal resection, particularly carinal pneumonectomy. Successful management of this lethal problem after pneumonectomy has been reported with the use of nitric oxide and may be applicable to carinal resection. ${ }^{15}$

We thank Robert H. Riffenburgh, $\mathrm{PhD}$, for his assistance in the preparation of this manuscript.

\section{REFERENCES}

1. Dartevelle P, Macchiarini P. Carinal pneumonectomy for bronchogenic carcinoma. Semin Thorac Cardiovasc Surg 1996;8:414-25.

2. Deslauriers J, Beaulieu M, McClish A. Tracheal sleeve pneumonectomy. In: Shields TW, editor. General thoracic surgery. 3rd ed. Philadelphia: Lea and Febiger; 1989. p. 382-7.

3. Roviaro GC, Varoli F, Rebuffat C, et al. Tracheal sleeve pneumonectomy for bronchogenic carcinoma. J Thorac Cardiovasc Surg 1994;107:13-8. 
4. Jensik RJ, Faber LP, Kirtle CF, et al. Survival in patients undergoing tracheal sleeve pneumonectomy for bronchogenic carcinoma. J Thorac Cardiovasc Surg 1982;84:489-96.

5. Grillo HC. Carinal reconstruction. Ann Thorac Surg 1982;34: 356-73.

6. Mathisen DJ, Grillo HC. Endoscopic relief of malignant airway obstruction. Ann Thorac Surg 1989;48:469-75.

7. Muehrcke DD, Grillo HC, Mathisen DJ. Reconstructive airway operation after irradiation. Ann Thorac Surg 1995;59:14-8.

8. El Baz N, Ganzouri AE, Gottschalk W, Jensik RJ. One lung highfrequency positive-pressure ventilation for sleeve pneumonectomy: an alternative technique. Anesth Analg 1981;60:683-6.

9. Montgomery WW. Suprahyoid release for tracheal stenosis. Arch Otolaryngol 1974;99:255-9.

10. Grillo HC, Mark EJ, Mathisen DJ, Wain JC. Idiopathic laryngotracheal stenosis and its management. Ann Thorac Surg 1993; 56:80-7.

11. Grillo HC, Shepard JO, Mathisen DJ, Kanarek DJ. Postpneumonectomy syndrome: diagnosis, management, and results. Ann Thorac Surg 1992;54:638-51.

12. Wright CD, Wain JC, Mathisen DJ, Grillo HC. Postpneumonectomy bronchopleural fistula after sutured bronchial closure: incidence, risk factors and management. J Thorac Cardiovasc Surg 1996;112:1367-71.

13. Mathisen DJ, Grillo HC. Carinal resection for bronchogenic carcinoma. J Thorac Cardiovasc Surg 1991;102:16-23.

14. Grillo HC, Mathisen DJ. Primary tracheal tumors: treatment and results. Ann Thorac Surg 1990;49:69-77.

15. Mathisen DJ, Kuo EY, Hahn C, et al. Inhaled nitric oxide for adult respiratory distress syndrome following pulmonary resection. Ann Thorac Surg. In press.

\section{Discussion}

Dr Jean Deslauriers (Sainte-Foy, Quebec, Canada). In the business of carinal surgery, resection is often straightforward but reconstruction can prove to be much more difficult. The low operative mortality rate and relatively low incidence of anastomotic complications achieved in this series, which includes a majority of complex reconstructions, is a tribute to the skills and judgment of the operating surgeons.

In patients with lung cancer, mediastinoscopy is recommended not only for staging purposes but also to help mobilize the trachea from above. How did the authors conciliate the need for maximal lymphadenectomy in patients who had cancer with the need for preservation of the lateral blood supply to the trachea? Perhaps the authors can also comment on the long-term fate of patients with lung cancer who underwent carinal resection.

Surgical access for lesions of the left main bronchus is controversial. In this series, 5 of the 8 patients, who either had a clamshell incision or bilateral same-day thoracotomies, required postoperative ventilatory assistance. For this reason we prefer a staged procedure wherein the first stage, a left proximal pneumonectomy, is performed accepting a positive resection margin. Three to 5 weeks later the carina is resected from the right side. Excellent exposure to the carina is also possible through a median sternotomy where a left pneu- monectomy and an intrapericardial release of either lung can be performed if required.

One of the technical problems associated with carinal resection is how much of the trachea can be safely resected. This is especially true on the left side where a tension-free anastomosis is difficult because of the anatomic relationship between the left main bronchus and the aorta. In this article, the authors rightly recommend that no more than $4 \mathrm{~cm}$ of airway be resected under such circumstances. Although neck flexion is the most important release maneuver in this situation, I would question the use of a chin to anterior chest wall stitch, especially in patients who are undergoing pneumonectomy. It is my impression that this flexed position favors aspiration and to some extent prevents productive coughing.

The surgical principles used for reconstruction of a healthy anastomosis (which include avoidance of technical errors, anastomotic tension, and ischemia) have been well presented today. If these principles are religiously followed, early dehiscence is uncommon, although local ischemia may result in late strictures rather than early disruption. Do the authors have any comments or data on the incidence of local recurrences after tumor resection?

Over the past few years, we have been very aware of the ARDS, which was seen in 10 of 124 cases presented in this report. Do the authors have any insight as to what causes this syndrome and how to prevent it? Our impression is that it has nothing to do with fluid overload but more to do with volutrauma to the lung and occult microaspiration.

Dr Mitchell. With respect to the question regarding lymphadenectomy, precisely because of concerns over airway devascularization, we do not do a complete mediastinal lymph node dissection in these patients but only remove those nodes immediately adjacent or clearly involved with the tumor.

With respect to the questions on the long-term fate of the patients with lung cancer and the question about local recurrence, because of time concerns with this presentation and space concerns with respect to the accompanying article, we did not include those data in this report; but we are currently analyzing those data and hope to report on them in the future.

With respect to the surgical access for left carinal pneumonectomy, we would agree that the idea of a staged left carinal pneumonectomy, which consists of a conventional left pneumonectomy leaving behind a knowingly positive bronchial stump margin, with an interval carinal resection through a right thoracotomy about 6 weeks later is an option in selected cases. In many of our patients, however, there was gross tumor involvement of the proximal left main bronchus that would have made the prospect of a secure bronchial stump closure uncertain.

We believe that a median sternotomy approach is acceptable for limited resections regarding left carinal pneumonectomy. It is also good for certain mid-level tracheal tumors and has been used for such in our institution. But again, we think it is not well suited for more extensive resections involving left carinal pneumonectomy. 
Regarding the question on neck flexion and a chin stitch, I think there is still some misconception about the degree of neck flexion maintained by a chin stitch as used at our institution. The neck is held in mild flexion. The primary reason for having the chin stitch is to prevent neck extension that would be detrimental to the anastomosis in the early postoperative period. We avoid extreme flexion of the neck.

With respect to the question about aspiration, we have not noted an increased incidence in aspiration in these patients or in any of the over 1000 tracheal resections performed at the Massachusetts General Hospital in which this same maneuver was used.
Finally, the development of ARDS is a devastating problem and was so in this series with a mortality rate of $90 \%$. Looking at this subgroup of patients, the one common thread was that, in 8 of the 10 patients, ARDS developed after carinal pneumonectomy. Several factors have been put forth as potential causes of ARDS (ie, fluid overload, interruption of lymphatics, complement activation, barotrauma); we agree that certainly fluid overload would not seem to be a likely cause, given the way these patients are now managed both during and after the operation. Our group recently reported some success in treating ARDS after lung resection using nitric oxide, and hopefully this success will extend to the carinal patients as well.

\section{Authoritative}

The Journal of Thoracic and Cardiovascular Surgery is the most frequently cited thoracic/cardiovascular surgery journal in the Science Citation Index. An article in JTCVS is sited on average almost twice as often as those in the closest cardiothoracic journal. 